\title{
Enabling compassionate healthcare: perils, prospects and perspectives
}

\author{
Russell Mannion*
}

Abstract

There is an emerging consensus that caring and compassion are under threat in the frenetic environment of modern healthcare. Enabling and sustaining compassionate care requires not only a focus on the needs of the patient, but also on those of the care giver. As such, threats and exhortations to health professionals are likely to have limited and perverse effects and it is to the organisational and system arrangements which support staff that attention should shift. Any approach to supporting compassionate care may work for some services, for some patients and staff, some of the time. No single approach is likely to be a panacea. Unravelling the contexts within which different approaches are effectual will allow for more selective development of support systems and interventions.

Keywords: Compassion, Healthcare Quality, Emotional Labour

Copyright: @ 2014 by Kerman University of Medical Sciences

Citation: Mannion R. Enabling compassionate healthcare: perils, prospects and perspectives. Int J Health Policy Manag 2014; 2: 115-117. doi: 10.15171/ijhpm.2014.34
Article History:

Received: 15 February 2014

Accepted: 3 April 2014

ePublished: 3 April 2014

\section{Correspondence to:}

Russell Mannion

Email: r.mannion@bham.ac.uk

\section{Introduction}

Major reforms of healthcare systems around the globe have focused on improving the quality and performance of healthcare services. Yet despite the scale of structural change, substantial additional resources and significant advances in treatment there remain concerns that in the fast paced and time pressured environment of modern healthcare, it is becoming increasingly difficult to respond with sensitivity and compassion to all patients all of the time. Nowhere is this more apparent than in the English National Health Service (NHS) where recent damning reports have exposed appalling standards of personal care and neglect at some hospitals and care homes $(1,2)$, with the national government advocating an urgent need for wholesale cultural change (3) and the nursing profession seeking a revitalisation of its core professional values and working practices to address the problem (4). Similar concerns have emerged in many other health systems, not least in the United States, where a recent national survey found that only $53 \%$ of patients and $58 \%$ of physicians felt the health system provides good levels of compassionate care (5). Many physicians in the survey indicated that changes in the healthcare system, in particular the emphasis on controlling costs, were preventing them from providing the level of care and emotional support to patients that they would want to give. Yet in any consideration of the role of compassion in healthcare, it is necessary not only to explain what is meant by compassion, but also to consider how compassion can be nurtured and sustained across a range of healthcare settings.

\section{The elements of compassionate healthcare}

In order to consider the key elements of compassion in healthcare it is useful to have a clearer idea of what compassion is. Compassion is a complex, contested and value laden concept fraught with rival interpretations and eluding a consensual definition. At the individual level compassion has variously been considered as an innate human quality, an evolutionary adaptation or an attitude of mind that can be acquired or taught $(6,7)$. According to the Oxford English Dictionary, compassion is derived from the ecclesiastical Latin stem compati, or to "suffer with". Although emphases differ, most approaches tend to view compassion as being closely linked with the ability to express empathy, provide emotional support and a willingness to understand and relieve another's distress and suffering. A recent narrative synthesis of the literature concluded that compassion in a healthcare context comprises four key elements (8):

- A relationship based on empathy, emotional support and efforts to understand and relieve a person's distress, suffering or concerns;

- Effective interactions between participants over time and across settings;

- Staff, patients and families being active participants in decision making; and

- Contextualised knowledge of the patient and family both individually and as members of a network of relationships.

For most health professionals compassion, empathy and respect are core values and viewed as integral to their caring role. However, there is growing evidence to support the view that compassionate care can also have a direct impact on health outcomes. The logic is that enhanced communication between a compassionate care giver and a patient helps build trust. This results in more shared information about symptoms and concerns which in turn yield a more accurate understanding and diagnosis. Compassionate care has also been linked to 
several other patient benefits including reduced anxiety and better adherence to treatment recommendations $(5,9)$.

\section{Overcoming barriers to compassion}

A lack of compassion, empathy or indifference to the suffering of others has been attributed to a number of factors including: the normal human defences and fears associated with having to cope with the burden of routinely being exposed to pain, distress and death on a daily basis; traditional pedagogic methods in medicine and nursing which focus on scientific neutrality and teach health professionals to maintain an emotional distance from patients; and the 'compassion fatigue' associated with caring jobs that entail significant stress and investments of emotional labour.

While it is tempting to blame poor practice and compassionless care on the individual doctor or nurse delivering care, we know that the organisational and cultural context can make an important difference in how care is delivered and experienced (10-12). There are a number of possible reasons for this. First, the ability of healthcare staff to demonstrate compassion may be as much a function of the levels of staffing in a particular ward (and hence the amount of time they have available for an individual patient), as it is the personality and predisposition of the caregiver. Second, factors in the wider care system may also be significant including the dysfunctional incentives associated with meeting external performance targets and, paradoxically, the lack of measures and incentives for compassion. Third, the priority given to the delivery of person-centred care in the front line of the service is influenced by the values, strategic priorities and leadership style at the top of the organisation. Modern healthcare arrangements thus pose challenges to the delivery of compassionate care. Shorter lengths of hospital stay, changing shift patterns resulting in shorter hand - over periods, the demands of external systems of checking, verification and audit and a proliferation of professional staff and agencies involved in care can hamper the development of personal, caring relationships and erode compassionate care.

\section{Supporting staff to provide care with compassion, dignity} and respect

Deficiencies in basic care arise in large part as an emergent outcome of a complex system where typically well-intentioned and competent staff interact in inadequate systems and unsupportive organisational environments (12). Poor health outcomes, unsafe care, lack of dignity, and compassionless care are emergent properties of these systems (although such a view does not, of course, absolve individuals from professional responsibility for the care that they provide). Therefore, compassion is not just an issue for frontline staff at the sharp end of care delivery. It is something that is accomplished and reproduced at all levels of the healthcare organisation, from 'bedside to Board'.

Compassionate care emerges from the routine enactment of behaviour, dialogue and decisions, much of which takes place far removed from frontline care delivery but which may nevertheless have a profound impact on the ability and motivations of frontline staff to behave in a compassionate way towards the patients in their care. Given the emotional burden and labour associated with caring it is unrealistic to assume that health professionals have an unlimited capacity for empathy, compassion, and emotional support towards each and every one of their patients, with perhaps stress and burnout affecting their ability to provide high quality care at all times (13). Therefore, enabling compassionate care requires not only a focus on the needs of the patient, but also on those of the care giver. As such, threats and exhortations to individual healthcare professionals working on the frontline of care delivery are likely to have limited (and sometimes perverse) effects, and it is to the organisational and system arrangements which support staff that attention should shift (14). In this regard, it is noteworthy that there are several initiatives to improve and support compassionate practice in healthcare. The Schwartz Center for Compassionate Care based in the United States and the Point of Care programme in the UK are currently at the forefront of international efforts to improve the experiences of patients and staff through a range of tailored organisational interventions underpinned by bespoke educational and training programmes.

\section{Concluding remarks}

There is an emerging view that compassion is under threat in the high volume, high risk world of modern healthcare. Yet compassion is a varied and complex phenomenon and what it means to be compassionate can be defined and interpreted in many different ways. Crucially though, compassionate care does not occur in a vacuum and organisational context is particularly pertinent in shaping and mediating how care is delivered, experienced and received. Any approach to supporting compassionate care may work for some services, for some patients and staff, some of the time. No single approach is likely to be a panacea. Different approaches might be needed in acute care compared with primary care and different supporting structures may be required for staff, patients, carers, and families. Unravelling the contexts within which different approaches are effectual will allow for more selective development of support systems and interventions. While we know much about how compassion is accomplished, there is much yet still to uncover with many gaps and inadequacies in the evidence base that need to be addressed. In particular, we need to deepen and broaden our understanding of the organisational processes and cultures which sustain (or erode) compassionate care across a range of healthcare settings and how compassion is linked to patient experience and health outcomes. These are important issues for health policy and management which merit further and more nuanced exploration, theoretically and empirically.

\section{Ethical issues Not applicable.}

\section{Competing interests \\ The author declares that he has no competing interests.}

\section{Author's contribution}

RM is the single author of the manuscript.

\section{References}

1. Abraham A. Care and compassion: Report of the health service ombudsman on ten investigations into NHS care of older people, fourth report if the health service commissioner for England; session 2010-2011, HC 778. London: The Stationary Office; 2011.

2. Francis R. Report of the Mid Staffordshire Foundation NHS Trust Public Inquiry Volumes 1-3, HC-898-I-III. London: The Stationery Office; 2013.

3. Davies $\mathrm{H}$, Mannion R. Will prescriptions for culture change improve the NHS? BMJ 2013; 346: f1305. 
4. Department of health. Developing the culture of compassionate care: creating a new vision and strategy for nurses, midwives and care givers. London: HSMC; 2012.

5. Lown B, Rosen J, Martilla J. An agenda for improving compassionate care: a survey shows about half of patients say such care is missing. Health Aff (Millwood) 2011; 30: 1772-8.

6. Cornwell J, Goodrich J. Exploring how to enable compassionate care in hospitals to improve patient experience. Nurs Times 2009; 105: 15-23.

7. Chochinov J. Dignity and the essence of medicine: the $a, b$ and $c$ and $d$ of dignity conserving care. BMJ 2007; 335: 184-7.

8. Dewar B, Nolan M. Caring about caring: developing a model to implement compassionate releationship ceneterd care in an older people care setting. Int J Nurs Stud 2013; 50: 1247-58.

9. Epstein RM, Franks P, Shields CG, Meldrum SC, Miller KN, Campbell TL, et al. Patient -centered communication and diagnostic testing. Ann Fam Med 2005; 3: 415-21.

10. Jacobs R, Mannion R, Davies HTO, Harrison S, Konteh F, Walshe K. The relationship between organizational culture and performance in acute hospitals. Soc Sci Med 2013; 76: 115-25

11. Mannion R, Davies HTO, Marshall M. Cultural attributes of 'high' and 'low' performing hospitals. J Health Organ Manag 2005; 19: 431-9.

12. Mannion R, Davies HTO, Marshall M. Cultures for Performance in Health Care. London: Open University Press; 2005.

13. Sawbridge $Y$, Hewsion A. Thinking about the emotional labour of nursing supporting nurses to care. J Health Organ Manag 2013; 27: 127-33.

14. Rynes SL, Bartunek JM, Dutton JE, Margolis JD. Care and compassion through an organizational lens: opening up new possibilities. Acad Manageme Rev 2012; 37: 503-23. 